\title{
La bioraffinerie comme objet transitionnel de la bioéconomie
}

\author{
Martino Nieddu et Franck-Dominique Vivien
}

\section{(2) OpenEdition}

Édition électronique

URL : http://journals.openedition.org/economierurale/4710

DOI : 10.4000/economierurale.4710

ISSN : 2105-2581

Éditeur

Société Française d'Économie Rurale (SFER)

Édition imprimée

Date de publication : 15 décembre 2015

Pagination : 7-11

ISSN : 0013-0559

Référence électronique

Martino Nieddu et Franck-Dominique Vivien, « La bioraffinerie comme objet transitionnel de la bioéconomie », Économie rurale [En ligne], 349-350 | septembre-novembre 2015, mis en ligne le 15 décembre 2017, consulté le 19 avril 2019. URL : http://journals.openedition.org/economierurale/4710 ; DOI : 10.4000/economierurale.4710 


\title{
INTRODUCTION
}

\section{La bioraffinerie comme objet transitionnel de la bioéconomie}

\author{
Martino NIEDDU, Franck-Dominique VIVIEN • Université de Reims Champagne-Ardenne, \\ laboratoire Regards
}

es trois papiers de ce dossier cherchent, chacun à leur façon, à cerner les contours de la dyLnamique d'exploration des usages des ressources agricoles, et de la forêt. Cette dynamique s'inscrit dans des stratégies de substitution des ressources fossiles. Ces stratégies cherchent à inverser une tendance historique issue des révolutions scientifiques et industrielles du XIX et du XX' siècle. En particulier, la montée en puissance de l'usage du pétrole au cours du XX' siècle a conduit au remplacement des intrants renouvelables en agriculture et des produits d'origine végétale ou animale pour l'énergie, les matériaux et la chimie, voire l'alimentation, par des produits fossiles ; ce que G. Allaire et B. Daviron' ont qualifié d'émergence d'une agriculture minière.

Néanmoins, cette tendance lourde ne doit pas faire oublier que l'agriculture et la forêt sont restées au cours du XXe siècle des fournisseurs non négligeables de l'industrie, notamment pour la chimie et l'énergie. Durant les années 1930 et jusqu'à la fin des années 1950, les Américains ont cherché à développer une chemurgy à base agricole (Finlay, 2004)², avec des acteurs significatifs - par exemple Henry Ford qui tente d'échapper au lobby de l'acier, en travaillant sur des carrosseries automobiles biosourcées. Et l'on trouve jusqu'au début des années 1960 des brevets sur des nylons à base biosourcée.

Giulio Natta, un grand chimiste qui a obtenu le prix Nobel de chimie pour ses travaux sur la production de polyéthylène et de polypropylène d'origine pétrolière, se réjouissait alors dans un petit ouvrage de vulgarisation sur les polymères que les progrès de la chimie des produits fossiles libèrent des terres agricoles pour nourrir les hommes. Mais la conjonction de l'émergence des excédents agricoles et l'opportunité offerte par le premier choc pétrolier ont conduit à réactiver les programmes de recherche sur le renouvelable à destination non alimentaire, qui vont connaître un nouveau cycle d'espérances (et de désillusions) dans la période 1975-1990, avant d'aboutir au début des années 2000 , à travers les programmes liés aux agrocarburants, à la mise en forme de l'objet bioraffinerie.

1. Allaire G., Daviron B. (2015). Les grandes transformations de l'agriculture, XVIII ${ }^{\mathrm{e}} \mathrm{XXI} \mathrm{X}^{\mathrm{e}}$ siècle. École-chercheurs INRA «Mobiliser les approches par les transitions dans la recherche sur les changements agricoles et alimentaires : pourquoi et comment? ?, Sète, mars 2015. Daviron B. (2014). Turning organic economies into mineral economies: Two centuries of oils and fats use and supply in France. European Social Science History Conference, Vienne, 36 p.

2. Finlay Mark R. (2004). Old Efforts at New Uses: A Brief History of Chemurgy and the American Search for Biobased Materials. Journal of Industrial Ecology, vol. 7, n 3-4, pp. 33-46. 


\section{INTRODUCTION}

Si l'on envisage la bioraffinerie, à la suite des travaux de sociologie des sciences, comme un objet intermédiaire, à la fois objet technique et concept organisateur, le terme même de bioraffinerie se réfère bien à un objet transitionnel ; l'ambition qu'elle décrit est celle de faire de l'agriculteur un « moléculteur » impliqué dans la substitution du carbone fossile par du carbone bio. Ce carbone bio aurait les mêmes usages et subirait les mêmes traitements de cracking, de décomposition en éléments simples qu'il faut purifier dans une chimie de base pour fournir une chimie de spécialité qui reconstituerait les produits issus des voies fossiles.

La mobilisation de la science qui découle de telles stratégies a pris la forme d'une knowledge based bioeconomy appelant à reformer l'intégration verticale entre agriculture, chimie, énergie et matériaux dans un nouveau secteur dédié. II nous semble nécessaire, au-delà des aspects technico-économiques, d'ouvrir un programme de travail en sciences sociales sur les évolutions conduisant à la formation de ce secteur spécifique, ainsi que sur la régulation de ses formes de fonctionnement et de ses frontières.

Car les tensions porteuses d'enjeux de régulation du secteur de la bioraffinerie sont de plusieurs ordres. (1) Il y apparaît que la construction d'une ère post-pétrole ne saurait être fondée sur la réduction de l'usage du carbone et une « société décarbonée ", mais sur l'intensification de I'usage de carbone bio. (2) Les sites industriels pourraient se structurer, comme cela a commencé, autour de produits importés de pays cherchant à tirer profit d'une nouvelle rente pétrolière, celle du pétrole vert, au même coût écologique que celui-ci, et autour du développement de bioraffineries portuaires, suivant en cela un modèle mimétique avec l'industrie pétrochimique. (3) Cette bioéconomie engendrerait une intensification de l'usage des ressources naturelles, et non une baisse de la pression des hommes sur les milieux, au risque d'alimenter ou renforcer des ruptures dans les espaces naturels ou cultivés. (4) Ce modèle mimétique pourrait s'étendre à la chimie qui en découlerait pour reproduire les produits existants : pour reprendre l'expression $d^{\prime}$ 'une start-up du secteur : " the same chemicals, the same products, just a lot greener $»^{3}$.

L'étude de la transition vers des systèmes soutenables emprunte généralement aux outils théoriques proposés sur les systèmes sectoriels d'innovation et de production (Malerba, 2012) ${ }^{4}$, qui mettent l'accent sur la formation de nouvelles bases de connaissances, et aux approches évolutionnistes en termes de perspective multi-niveaux du Sustainability Transition Management

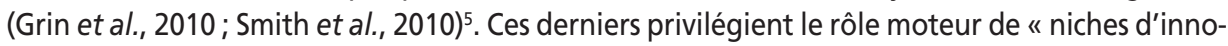
vations " pour explorer de nouveaux régimes socio-techniques. Dans une perspective régulationniste, d'autres insistent sur les dynamiques de longue période de l'action collective - avec des " acteurs situés " qui s'affrontent et cherchent à construire des compromis institutionnalisés au sein desquels ils seront hégémoniques. D'où l'attention portée, en approche régulationniste, aux patrimoines productifs collectifs dont ils sont porteurs et qu'ils cherchent à projeter dans le futur (Nieddu, Vivien, 2014).

3. http://www.myriant.com/our-compagny/index.cfm, consulté le 13 mars 2014.

4. Malerba F., Nelson R. R. (dir.) (2012). Economic Development as a Learning Process: Variation Across Sectoral Systems. Cheltenham, Edward Elgar.

5. Grin J., Rotmans J., Schot J. (in collaboration with Frank Geels and Derk Loorbach) (2010). Transitions to Sustainable Development. London, Routledge, 381 p. ; Smith A., Voß J.-P., Grin J. (dir.) (2010). Innovation studies and sustainability transitions. Research Policy, Special Section on Innovation and Sustainability Transitions, vol. 39, $\mathrm{n}^{\circ} 4$.

6. Nieddu M., Vivien F.-D. (2014). Quels apports de l'approche patrimoniale pour l'analyse des transitions vers le développement soutenable : I'exemple de la chimie verte. Économie appliquée, vol. $67, n^{\circ} 4$, pp. 40-70. 
Ces patrimoines sont d'abord des ressources immatérielles. La construction des « visions pour le futur » et la constitution en « objets intermédiaires » de dispositifs techniques tels que la bioraffinerie sont autant d'outils cognitifs partagés, qui organisent les conditions d'apprentissage entre utilisateurs et producteurs. II s'agit ensuite d'institutions dédiées à la constitution en communauté, tels que les « plates-formes technologiques » de l'Europe ou les pôles de compétitivité en France. II s'agit donc d'un enjeu des stratégies des acteurs dans les processus de qualification - ou d'invalidation - de ressources comme patrimoines pour ce secteur, au moment de l'élaboration de feuilles de route technologiques par les institutions qui vont soutenir la recherche et le développement industriel. Au plan matériel, il s'agit de dispositifs « localisés » qui permettent aux acteurs scientifiques et économiques de se rencontrer : des laboratoires publics ou privés dédiés, des centres de développement technologique, des démonstrateurs, des unités pilotes avant industrialisation.

Les trois articles présentés dans ce dossier explorent certains aspects de ces dynamiques. Celui de Bente Hessellund Andersen et Michael Søgaard Jørgensen (du Center for Design, Innovation and Sustainable Transition, d'Aalborg University au Danemark) explore l'arène de développement danoise. Les « plans climat » danois intègrent la bioénergie dans leur feuille de route. Mais celle-ci est marquée par un fort phénomène de path dependency aux acteurs. En particulier, les auteurs notent la place qu'occupe une multinationale danoise, qui est un des leaders mondiaux dans les enzymes destinées aux productions de biocarburants (Novozymes pour ne pas la nommer), et celle de l'élevage industriel qui trouve une nouvelle source de revenus dans la production de biogaz et une nouvelle source d'arguments pour se renforcer.

L'article de Les Levidow (Open University, Royaume-Uni), qui a produit un ensemble de travaux sur les tensions entre les stratégies agro-écologiques et celles dites corporate-environmental, analyse l'agenda de la formation de la bioéconomie au niveau européen. II insiste sur le fait que cette bioéconomie cherche à s'inscrire dans les mêmes produits et à mobiliser les mêmes leviers que dans l'actuel régime de consommation. Il interroge également les visions du futur que portent les grandes institutions comme le World Economic Forum (WEF) ou la Commission européenne. Pour le premier, la bioraffinerie construit une nouvelle division du travail entre les pays disposant de grands espaces agricoles et les futurs clients de ceux-ci pour la biomasse et ses dérivés. Le WEF considère la bioéconomie comme une opportunité, pour les pays du Sud qui sont dans ce cas, de moderniser leurs pratiques agricoles et de sortir de l'agriculture de petites exploitations familiales, pour aller vers des systèmes de plantation intensifs.

Clarisse Cazals chercheure en économie patrimoniale et Arnaud Sergent chercheur en sciences politiques (tous deux à l'unité ADBX de I'Irstea, Bordeaux) proposent une revue de littérature sur les bioraffineries liées à l'industrie papetière associées à la bioéconomie. En effet, l'industrie papetière présente les mêmes caractéristiques que l'industrie alimentaire (utilisation d'une biomasse, saturation des marchés traditionnels, existence d'un patrimoine productif à préserver et projeter dans le futur). Celle-ci s'est donc d'autant plus emparée de la problématique de la bioraffinerie qu'elle suit un modèle de développement consistant « à ajouter des briques " à ses complexes industriels déjà installés, soit pour optimiser ses sites en valorisant ses déchets dans la bioraffinerie (tels que les liqueurs noires), soit pour ouvrir de nouveaux fronts de production, du fait de la pression à développer une bioraffinerie " de deuxième génération " (i.e. s'appuyant sur des substrats non alimentaires), à partir de l'hypothèse, rarement discutée, d'une sous-utilisation des forêts. Les auteurs, dans un travail qui se veut exploratoire, se proposent d'étudier, dans une perspective d'économie politique institutionnelle, la façon dont les acteurs problématisent la construction de leur industrie et sa place dans la transition écologique. 
L'originalité de la démarche tient donc dans le fait d'aborder les questions d'économie industrielle et $d$ 'innovation à travers ce prisme d'économie politique qui replace les actions collectives des acteurs, leurs patrimoines et leurs visions du futur au cœur des dynamiques observées.

Ces trois articles contribuent donc à dessiner l'espace des tensions dans la transition, à partir de l'objet transitionnel bioraffinerie, vers l'usage des ressources renouvelables. Ils suggèrent également le fait que la description des espaces de controverses permet de discuter la littérature du Sustainability Transition Management qui se représente la dynamique de formation de nouveaux régimes socio-techniques à partir de l'activité de niches d'innovation environnementale, et la contribution de ces niches à la définition d'un dominant design. On notera plutôt I'intensité de l'activité d'entrepreneuriat institutionnel d'acteurs bien établis et la construction de visions pour le futur, qui sont autant d'outils de pilotage des politiques scientifiques et industrielles (du type partenariats public-privé de la Commission européenne).

Ce qui renvoie à deux enjeux qui appellent un programme de travail en sciences sociales sur la bioraffinerie. Le premier est celui des produits issus de celle-ci. Dans une stratégie de substitution terme à terme des produits issus du fossile, il n'y a pas d'évolution attendue du côté des comportements de consommation. Il suffit de pouvoir qualifier les produits issus du renouvelable du point de vue de leur contenu en carbone bio ${ }^{7}$. Le deuxième enjeu est celui de l'ancrage territorial des activités liées à la bioéconomie. Levidow évoque un scénario de division internationale du travail où, comme dans la pétrochimie, une bioraffinerie portuaire traiterait les ressources en biomasse importées de pays disposant d'un avantage comparatif sur l'éthanol (Brésil) ou l'huile de palme (Malaisie). On aurait pu penser, au regard des exercices de prospective menés pour dessiner des feuilles de route technologiques par les grandes institutions, que les autres patrimoines productifs collectifs s'éteindraient à mesure que le mimétisme avec les formes d'organisation des chaînes de valeur de la pétrochimie se mettrait en place. Or on peut constater que ce mouvement n'a pas eu lieu. Les sites des industries alimentaires et de la papeterie restent la base de l'essentiel des développements en bioéconomie.

L'intensification de l'usage de la biomasse peut donc emprunter des voies différentes, et il est possible de poser la question de l'ancrage territorial des industries la transformant sur des ressources locales : la nature de ces ressources commande la pertinence des choix technologiques et les échelles auxquelles il est possible de porter l'outil de production, en respectant les caractéristiques de ces territoires agricoles pour ne pas exercer de pressions trop fortes sur l'environnement. Se pose alors la question d'un cadre institutionnel qui, a contrario des normes de concurrence, permettrait à des zones de productivité différentes de porter des voies technologiques à divers niveaux de rentabilité.

De ce point de vue, le constat dressé par la recherche, d'une variété de patrimoines productifs collectifs, et non d'un paradigme unique (Nieddu, Garnier, Bliard, 2014) ${ }^{8}$, invite à une exploration localisée de chacun des patrimoines productifs collectifs. Par exemple, la voie, typique de la pétrochimie et des procédés Fischer-Tropsch pour la biomasse, d'une " complète dégradation de la biomasse en petites molécules (dites (1) en building blocks par gazéification ou

7. Ce que fait la norme américaine ASTM 6866. Elle propose une datation au carbone 14 de l'âge du carbone utilisé dans le produit pour en établir le caractère biosourcé. Car, comme le produit est, du point de vue du chimiste, rigoureusement identique, c'est la seule solution pour identifier le contenu en carbone renouvelable d'un produit (tel que le contenu en éthylène glycol biosourcé de la bouteille en polyéthylène téréphtalate " vert » de Coca-Cola...).

8. Nieddu M., Garnier E., Bliard C. (2014). Patrimoines productifs collectifs versus explorationexploitation: le cas de la bioraffinerie. Revue économique, vol. 67, nº 6, pp. 957-987. 
méthanisation " (Marquardt et al., 2010) ${ }^{9}$ peut être la seule façon de traiter efficacement et localement des déchets agricoles ou urbains. Mais les autres patrimoines productifs collectifs peuvent être valorisés dans d'autres voies et permettre d'envisager des échelles de production variées. Comme il est certain que la bioéconomie et la bioraffinerie s'inscrivent dans un nouveau champ de contradictions ou de tensions entre intensification et dimensions environnementales et sociales des territoires, l'exploration de cette variété de patrimoines en bioraffinerie mérite d'être posée.

9. Marquardt W., Harwardt A., Hechinger M., Kraemer K., Viell J., Vollet A. (2010). The Biorenewables Opportunity. Toward Next Generation Process and Product Systems. AlChE, September, vol. $56, n^{\circ} 9$. 